\title{
Melanoma expression of matrix metalloproteinase-23 is associated with blunted tumor immunity and poor responses to immunotherapy
}

Duane Moogk $k^{1,2}$, Ines Pires da Silva ${ }^{3,4,5,6}$, Michelle W Ma ${ }^{3,4}$, Erica B Friedman ${ }^{4,7}$, Eleazar Vega-Saenz de Miera ${ }^{3,4}$, Farbod Darvishian 2,4, Patrick Scanlon ${ }^{3,4}$, Arianne Perez-Garcia, 1,2, Anna C Pavlick 1,3,4, Nina Bhardwaj 1,4,8, Paul J Christos ${ }^{9}$, Iman Osman ${ }^{1,3,4}$ and Michelle Krogsgaard ${ }^{1,2,4^{*}}$

\begin{abstract}
Background: Matrix metalloproteinase-23 (MMP-23) can block the voltage-gated potassium channel Kv1.3, whose function is important for sustained $\mathrm{Ca}^{2+}$ signaling during $\mathrm{T}$ cell activation. MMP-23 may also alter $\mathrm{T}$ cell activity and phenotype through cleavage of proteins affecting cytokine and chemokine signaling. We therefore tested the hypothesis that MMP-23 can negatively regulate the anti-tumor T cell response in human melanoma.

Methods: We characterized MMP-23 expression in primary melanoma patients who received adjuvant immunotherapy. We examined the association of MMP-23 with the anti-tumor immune response - as assessed by the prevalence of tumor-infiltrating lymphocytes and Foxp3 ${ }^{+}$regulatory T cells. Further, we examined the association between MMP-23 expression and response to immunotherapy. Considering also an in trans mechanism, we examined the association of melanoma MMP-23 and melanoma Kv1.3 expression.

Results: Our data revealed an inverse association between primary melanoma MMP-23 expression and the anti-tumor T cell response, as demonstrated by decreased tumor-infiltrating lymphocytes (TIL) $(P=0.05)$, in particular brisk TILs $(P=0.04)$, and a trend towards an increased proportion of immunosuppressive Foxp $3^{+}$regulatory $T$ cells $(P=0.07)$. High melanoma MMP-23 expression is also associated with recurrence in patients treated with immune biologics $(P=0.037)$ but not in those treated with vaccines $(P=0.64)$. Further, high melanoma MMP-23 expression is associated with shorter periods of progression-free survival for patients receiving immune biologics $(P=0.025)$. On the other hand, there is no relationship between melanoma MMP-23 and melanoma Kv1.3 expression ( $P=0.27$ ).
\end{abstract}

Conclusions: Our data support a role for MMP-23 as a potential immunosuppressive target in melanoma, as well as a possible biomarker for informing melanoma immunotherapies.

Keywords: Matrix metalloproteinase-23, Melanoma, Immunotherapy, Kv1.3, Tumor-infiltrating lymphocytes

\footnotetext{
*Correspondence: Michelle.Krogsgaard@nyumc.org

${ }^{1}$ Perlmutter Cancer Center at NYU Langone, New York, NY, USA

2Department of Pathology, New York University School of Medicine, New York, NY, USA

Full list of author information is available at the end of the article
}

\section{() Biomed Central}

(c) 2014 Moogk et al.; licensee BioMed Central Ltd. This is an Open Access article distributed under the terms of the Creative Commons Attribution License (http://creativecommons.org/licenses/by/4.0), which permits unrestricted use, distribution, and reproduction in any medium, provided the original work is properly credited. The Creative Commons Public Domain Dedication waiver (http://creativecommons.org/publicdomain/zero/1.0/) applies to the data made available in this article, unless otherwise stated. 


\section{Background}

Melanoma is a highly immunogenic tumor [1], yet tumor progression nevertheless occurs in immunocompetent patients, which suggests the existence of immune-regulatory mechanisms within the tumor. Tumors can evade immunemediated destruction through the release of soluble factors that redirect the immune response as well as via mechanisms that limit or inhibit the infiltration or the function of tumor-infiltrating lymphocytes (TILs) [1-3]. Many therapeutic strategies for melanoma have therefore been developed to augment anti-tumor immunity by targeting immunosuppressive mechanisms. Treatments aimed at these mechanisms, such as cytotoxic $\mathrm{T}$ lymphocyte-associated antigen-4 (CTLA-4) and programmed cell death 1 (PD-1), work to unrestrain pre-existing TILs from immunosuppressive checkpoints [1]. Select subsets of patients respond favorably to immune-based therapies, but given the morbidity associated with these treatments, clinicopathological criteria are needed to better identify those patients who could benefit and to optimize their immunotherapeutic strategy. Identification of new modulators of immune resistance may also lead to development of anti-melanoma therapeutics that are favorable to patients that are unresponsive to other treatments, or that may act as adjuvants to complement existing therapies to further improve patient outcomes.

Matrix metalloproteinases (MMPs) are a family of zincand calcium-dependent proteolytic enzymes that may be either membrane anchored or secreted [4]. The major function of MMPs is degradation of extracellular matrix (ECM) components [5], which can play a role in cancer progression by promoting tumor growth, infiltration and angiogenesis [6]. All MMPs share the common features of an N-terminal signal peptide that directs it to the secretory pathway, a catalytic domain containing a zinc ion in the active site, and a prodomain that interacts with the active site to block enzymatic activity until its removal [4,7]. MMPs also function to cleave non-matrix proteins, including surface receptors, and to activate chemokines and cytokines [4] - mechanisms that have been implicated in a number of other diseases, including arthritis, vascular disease, and Alzheimer's disease [7,8].

MMP-23 is a membrane-anchored MMP, distinguished from other MMPs in that its N-terminal pro-domain (MMP-23-PD) lacks the enzymatic inhibitory sequence and the characteristic C-terminal hemopexin domain is replaced by an immunoglobulin-like cell adhesion molecule domain [4]. Full-length MMP-23 is found predominantly in perinuclear and endoplasmic reticulum (ER) membranes $[9,10]$, and a single cleavage results in removal of the MMP-23-PD, activation and secretion from the cell [10]. Prior to cleavage, the MMP-23-PD may interact with Kv1.3 potassium channels and regulate their surface expression [11]. Further, MMP-23 also contains a toxin-like domain (MMP-23-TxD) immediately following the catalytic domain [12], which, upon secretion of active MMP-23, may block Kv1.3 channels on proximal cells [11]. MMP-23 therefore has the ability to interact with Kv1.3 in two distinct mechanisms that may affect Kv1.3 membrane expression or function.

The physiological effects of blocking Kv1.3 channels on autoreactive $\mathrm{T}$ cells have been demonstrated in the context of autoimmune diseases, including rheumatoid arthritis, type-1 diabetes mellitus, and multiple sclerosis $[13,14]$, where selective blocking may reduce unwanted autoimmune responses. Therefore, MMP-23 has the potential to affect cellular processes through in cis Kv1.3 trapping, in trans extracellular Kv1.3 blocking [11,12], or through cleavage of proteins affecting cytokine and chemokine signaling [4]. However, it is unclear if MMP-23 expression in melanoma or other cancers can affect disease progression through these mechanisms.

The focus on tumor MMPs in cancers, including melanoma, breast, prostate, lung, and colon cancer, has generally been on their ability to mediate microenvironmental changes to the ECM that regulate cancer progression [15-17]. However, MMPs also play a role in the regulation of anti-tumor immune responses [6]. For example, MMP cleavage of necrosis factor-alpha promotes NF- $\mathrm{k} \beta$ signaling, resulting in recruitment of immune cells [18]. Similarly, MMPs can influence $\mathrm{T}$ cell phenotype - active MMP-2 induces Th2 skewing by blocking IL-12 and inducing OX40L on dendritic cells [19]. While not generally used as a diagnostic marker of cancer, MMP expression has been widely studied as a potential prognostic marker for a number of cancers [7]. Increased tumor expression of MMP-1, MMP-2, MMP-7, or MMP-9 in lung cancers [20-22], and increased expression of MMP-1 and MMP-9 in breast cancer $[23,24]$ is associated with poor patient survival. MMPs, therefore, may be suitable as both biomarkers for cancer prognosis and as therapeutic targets. In melanoma, increased expression of MMP-2 is associated with high invasiveness and melanoma progression [25,26], while expression of MMP-9 was associated with metastasis [27]. However, inhibitors designed for general targeting of MMPs have not seen clinical success, and have lead to a number of undesirable side effects [28], highlighting the need for the development of inhibitors with specific targeting properties.

In this study, we characterize for the first time MMP-23 expression in human melanomas as it relates to antitumor immunity and clinical response to immunotherapy. Our data support a role for MMP-23 in blunting the anti-tumor response. Further, our data also support a role for MMP-23 in diminishing melanoma patient responses to immune biologic immunotherapies. This highlights the potential use of MMP-23 melanoma expression as a predictive biomarker for the selection of 
therapeutic adjuvants and as a potential therapeutic target.

\section{Methods \\ Research design}

Primary melanoma tissues obtained before the start of immunotherapy were retrieved from patients enrolled in the Interdisciplinary Melanoma Cooperative Group, a prospectively collected clinicopathologic-biospecimen database at New York University Medical Center [29], between August 2002 and December 2008. Patients were treated with immune-based therapeutics after primary resection or at recurrence. Immunotherapies were categorized as immune biologics (IFN- $\alpha$, IL-2, GM-CSF) or vaccines (dendritic cell-, peptide-). Informed consent was obtained from all patients at the time of enrollment. Demographic and clinicopathologic information collected included age at pathological diagnosis, gender, primary tumor thickness (mm), ulceration status, mitosis (absent vs. present), histotype, anatomic site, TILs (absent vs. present: non-brisk, brisk as identified by characteristic lymphocytic morphology on hematoxylin-and-eosin staining) [30], recurrence status, and melanoma status at last follow-up (December 2010). TILs were defined as brisk when present throughout the vertical growth phase (i.e. large dermal aggregates of melanoma over 15-25 cells wide) or present and infiltrating across the entire base of the vertical growth phase [30]. All research was approved by the NYU School of Medicine's Office of Science and Research Institutional Review Board ("Development of an NYU Interdisciplinary Melanoma Cooperative Group: A Clinicopathological Database", IRB Study \# i10362).

\section{Immunohistochemical analysis}

Immunohistochemistry was performed using rabbit polyclonal anti-human MMP-23 - carboxyterminal end (ab39087, Abcam, Cambridge, MA, USA), anti-Kv1.3 (APC-002, Alomone Labs, Ltd., Jerusalem, Israel), and mouse monoclonal anti-human Foxp3 (clone 236A/E7) (eBioscience, San Diego, CA, USA) antibodies on formalinfixed, paraffin-embedded primary melanoma tissues to detect MMP-23 expression by melanoma cells, Kv1.3 potassium channel expression on tumor cells, and Foxp $3^{+}$ $\mathrm{T}_{\text {regs }}$, respectively. In brief, after deparaffinization and rehydration, heat-induced epitope retrieval for MMP-23, Kv1.3, and Foxp3 were performed in $0.01 \mathrm{M}$ citrate buffer, $\mathrm{pH} 6.0$, in a 1,200 -watt microwave oven at $100 \%$ power for 20,10 , and 10 minutes, respectively. Sections were cooled in tap water for 5 minutes, quenched in $0.3 \%$ hydrogen peroxide for 30 minutes, washed with PBS, and incubated for 30 minutes with diluted normal blocking serum prepared from goat serum for MMP-23 and horse serum for Foxp3 while a blocking solution containing 5\% bovine serum albumin, $0.1 \%$ sodium azide, and $5 \%$ goat serum was used for Kv1.3. Slides were then incubated with each primary antibody diluted in buffer (MMP-23, 1:100; Kv1.3, 1:50; Foxp3, 1:500) at room temperature for 1 hour and at $4^{\circ} \mathrm{C}$ overnight, after which they were washed in buffer and incubated with diluted biotinylated secondary antibodies (goat anti-rabbit at 1:500 for both MMP-23 and Kv1.3-stained sections; horse anti-mouse at 1:500 for Foxp3-stained sections, Vector Laboratories, Burlingame, CA, USA) for 1 hour. Avidin-biotinylated horseradish peroxidase complexes diluted at 1:500 ( $\mathrm{ABC}$ reagent, Vector Laboratories) were added. MMP-23 and Kv1.3 staining were both visualized with peroxidase ImmPACT $^{\mathrm{mm}}$ NovaRED $^{\mathrm{mm}}$ Peroxidase Substrate, Vector Laboratories) and diaminobenzidine (DAB substrate kit, Vector Laboratories) was used to visualize Foxp3 staining. Sections were washed in distilled water, counterstained with hematoxylin, dehydrated, and then mounted with permanent media. Appropriate positive and negative controls were included with study sections as well. Specificity of the MMP23 antibody was shown by competition experiment with immunizing peptide (Abcam ab41122) (Additional file 1: Figure S1). MMP23 antibody at a dilution of $1 \mu \mathrm{g} / \mathrm{ml}$ was pre incubated with $2 \mu \mathrm{g} / \mathrm{ml}$ of immunizing peptide for $1 \mathrm{~h}$ at room temperature before the application to the tissue, as described above. MMP23 antibody was also assessed by Western blot using $10 \mu \mathrm{g}$ of protein extracted from melanoma tissues, melanoma cell line, or placenta as positive control, and probed with anti-MMP-23 antibody at 1:5000 dilution (Additional file 2: Figure S2).

An attending pathologist (F.D.) blinded to all clinical data scored the slides for MMP-23, Foxp3, and Kv1.3 expression. Tumor MMP-23 expression was scored for staining intensity $(0=$ none, $1=$ faint, $2=$ intense, $3=$ very intense $)$ and distribution $(0=$ none, $1=$ focal $(<50 \%), 2=$ diffuse $(\geq 50 \%)$ ), which were summed to generate a composite score for each case as illustrated in Figure 1. Foxp3 expression was scored as the absolute number of positively stained cells with characteristic lymphocytic morphology in a representative high-power field $\left(0.2 \mathrm{~mm}^{2}\right)$, and Kv1.3 expression was scored as the absence or presence of Kv1.3 staining on melanoma cells. Each representative highpower field was selected by scanning each slide at 100x to identify the field with the highest antibody expression.

\section{Statistical analysis}

Descriptive statistics were calculated for MMP-23 expression, Kv1.3 expression, and clinicopathologic variables. Univariate associations between MMP-23 expression and continuous clinicopathologic variables were assessed by the ANOVA test or Kruskal-Wallis test, as appropriate. Univariate associations between MMP-23 expression and categorical clinicopathologic variables (including recurrence) were assessed by the chi-square test or Fisher's exact test, as appropriate. Progression-free survival was defined as the time from immunotherapy to recurrence. Event-time 


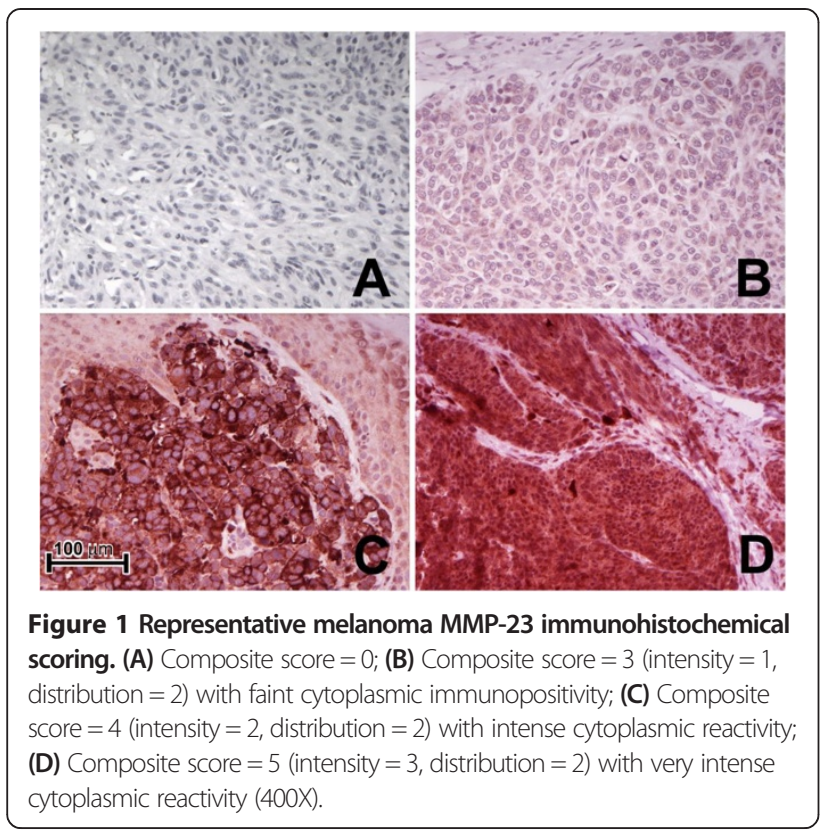

distributions were estimated with the use of the KaplanMeier method. Categories of high and low MMP-23 expression were explored in various analyses. All p-values are two-sided with statistical significance evaluated at the 0.05 alpha level. All analyses were performed in SPSS Version 21.0 (SPSS Inc., Chicago, IL).

\section{Results}

\section{Patient selection and treatment}

Table 1 illustrates the demographic and clinicopathological characteristics of the cohort of primary melanoma patients studied. Primary melanoma specimens acquired prior to the initiation of immunotherapy were examined for each of these patients $(n=71)$. Immunotherapy was given after primary resection $(n=40)$ or at recurrence $(\mathrm{n}=31)$ (Table 1). Patients were treated with a variety of immunotherapies, categorized as immune biologics $(n=38$; IFN- $\alpha$, IL-2, GM-CSF) or vaccines $(\mathrm{n}=33$; dendritic cell-, peptide-). Informed consent was obtained from all patients at the time of enrollment. Median follow-up time from the date of pathological diagnosis was 6.3 years (range: 1-10 years). Melanoma was the cause of death for $38 / 39$ patients who died during follow-up.

\section{Melanoma MMP-23 expression does not correlate with melanoma Kv1.3 expression}

Tumor-derived MMPs in melanoma and other cancers can mediate microenvironmental changes regulating cancer progression [15-17]. Melanoma cells can express Kv1.3 [31], although the role of tumor Kv1.3 expression is unclear. However, in prostate cancer, reduced tumor cell Kv1.3 expression is associated with poor clinical outcome [32]. We therefore hypothesized that melanoma Kv1.3
Table 1 Demographic and clinicopathologic characteristics of melanoma patients treated with immunotherapy

\begin{tabular}{ll}
\hline Variable & Number of patients $(\mathbf{n}=\mathbf{7 1})$ \\
\hline Age at diagnosis (years) & \\
$\quad$ Median (Range) & $55(21-80)$ \\
Gender & \\
$\quad$ Male & $40(56.3 \%)$ \\
$\quad$ Female & $31(43.7 \%)$ \\
AJCC stage at diagnosis & \\
I & $5(7.1 \%)$ \\
II & $26(36.6 \%)$ \\
III & $40(56.3 \%)$
\end{tabular}

Primary TILs

$\begin{array}{cl}\text { Absent } & 16(22.6 \%) \\ \text { Present } & 52(73.2 \%) \\ \text { Non-brisk } & 29 \\ \text { Brisk } & 23 \\ \text { Unclassified } & 3(4.2 \%)\end{array}$

Immunotherapy setting ${ }^{a}$

$\begin{array}{ll}\text { Adjuvant } & 40(56.3 \%) \\ \text { At Recurrence } & 31(43.7 \%)\end{array}$

Type of immunotherapy

$\begin{array}{cl}\text { Immune biologic } & 38 \text { (53.5\%) } \\ \text { IFN-a } & 21 \\ \text { IL-2, IL-18 } & 4 \\ \text { GM-CSF } & 12 \\ \text { Other } & 1 \\ \text { Vaccine } & 33(46.5 \%) \\ \text { Dendritic cell } & 11 \\ \text { Peptide } & 22\end{array}$

Abbreviations: AJCC American joint committee on cancer, TILs Tumor-infiltrating lymphocytes, NOS Not otherwise specified.

a Patients who received immunotherapy in both settings $(n=6)$.

surface expression may be inhibited by in cis MMP-23 trapping of Kv1.3 at the ER [12]. To examine the possible effect of melanoma MMP-23 expression on tumor Kv1.3 expression, 20 primary melanomas were evaluated for both MMP-23, as measured by a composite score of MMP-23 staining intensity and distribution (Figure 1), and Kv1.3 expression. Kv1.3 expression was absent in $10 / 15$ (67\%) primary tumors that had high MMP-23 expression as well as in 5/5 (100\%) primary melanomas that had absent or low MMP-23 expression $(P=0.27)$ (Table 2). Our data therefore suggest that tumor MMP-23 expression does not directly affect tumor Kv1.3 expression as would occur via an in cis mechanism. Our data did not demonstrate an association between tumor Kv1.3 expression and clinical outcome as assessed by recurrence following 
Table 2 MMP-23 expression and recurrence according to Kv1.3 expression.

\begin{tabular}{|c|c|c|c|c|c|c|}
\hline \multirow[b]{2}{*}{ Kv1.3 expression } & \multicolumn{3}{|c|}{ MMP-23 composite score ${ }^{a}$} & \multicolumn{3}{|c|}{ Recurrence $^{\mathbf{b}}$} \\
\hline & $0-2$ & $3-4$ & & Yes & No & \\
\hline Yes & $0(0 \%)$ & $5(33 \%)$ & $5(100 \%)$ & $4(27 \%)$ & $1(20 \%)$ & $5(100 \%)$ \\
\hline No & $5(100 \%)$ & $10(67 \%)$ & $15(100 \%)$ & $11(73 \%)$ & $4(80 \%)$ & $15(100 \%)$ \\
\hline Total & $5(100 \%)$ & $15(100 \%)$ & & 15 (100\%) & $5(100 \%)$ & \\
\hline
\end{tabular}

$\mathrm{a} \mathrm{P}=0.27 ;{ }^{\mathrm{b}} \mathrm{P}=0.99$.

immunotherapy. Recurrence was observed in 11/15 (73\%) patients whose primary tumors were absent Kv1.3 expression, and similarly in $4 / 5(80 \%)$ patients whose primary melanomas stained positive for Kv1.3 $(P=0.99)$ (Table 2). Therefore, there does not appear to be a link between melanoma MMP-23 and Kv1.3 expression, and further, melanoma Kv1.3 expression does not correlate with immunotherapy outcome.

Higher melanoma MMP-23 expression is associated with a blunted anti-tumor immune response

In the absence of in cis regulation of melanoma Kv1.3, MMP-23 also has the potential to act in trans through cleavage of surface protein or blocking Kv1.3 channels on nearby cells, including TILs. Blocking of Kv1.3 on T cells can diminish the driving force for calcium influx, thereby reducing activation-induced proliferation and motility [33]. Furthermore, prolonged inhibition of Kv1.3 can prevent $\mathrm{T}$ cells from receiving activation or survival signals, resulting in death due to cytokine deprivation [14]. Cleavage of non-ECM proteins may affect cytokine and chemokine signaling, and has the potential to skew $\mathrm{T}$ cell phenotype $[18,19]$. We therefore considered that melanoma MMP-23 expression could have a role in regulating anti-tumor immune responses.

To evaluate the relationship between primary melanoma MMP-23 expression and anti-tumor immunity, we compared melanoma MMP-23 expression with two separate measures of the strength of the intrinsic anti-tumor immune response - the presence and the degree of TIL infiltration [30] - at the time of primary resection (Figure 2). We observed that increased melanoma MMP-23 expression is inversely associated with the presence of TILs (presence $=79.4 \%$ for melanomas with low MMP-23 expression and $53.8 \%$ for melanomas with high MMP23 expression, $P=0.05$ ). Quantification of the intensity of lymphocytic infiltration also showed a significant inverse correlation between MMP-23 expression and a brisk lymphocytic response (brisk TILs $=65.0 \%$ for melanomas with low MMP-23 expression and $25.0 \%$ for melanomas with high MMP-23 expression, $P=0.04$ ). These results suggest a role for tumor-derived MMP-23 in the suppression of anti-tumor immune responses.
In addition to tumor-specific T-lymphocytes, the TIL population is also comprised of immunosuppressive Foxp $3^{+}$ regulatory $T$ cells $\left(\mathrm{T}_{\text {regs }}\right)$ that play an important role in immune evasion $[34,35]$. The accumulation of $T_{\text {regs }}$ in the tumor microenvironment can be attributed to a number of factors, including the local expression and secretion of factors affecting $\mathrm{T}_{\text {reg }}$ migration and retention, the expansion of naturally occurring $\mathrm{T}_{\text {regs }}$, or the de novo generation of induced $\mathrm{T}_{\text {regs }}$ [36]. To investigate the potential role of melanoma MMP-23 in contributing to conditions favorable to $\mathrm{T}_{\text {regs }}$, we assessed the relationship between MMP-23 expression and $\mathrm{T}_{\text {reg }}$ prevalence, as determined by the number of Foxp $3^{+}$cells (Figure 3 ). We observed a trend towards an increased number of $\mathrm{T}_{\text {regs }}$ in primary melanomas with higher MMP-23 expression (53.1 \pm 33.8 in melanomas with high MMP-23 expression and $35.0 \pm 25.1$ in melanomas with low MMP-23, $P=0.07)$. This suggests a potential role for MMP-23 in skewing TIL phenotype. Combined, these results suggest that MMP-23 plays a role in blunting the immune response to melanoma as it affects the prevalence, distribution, and composition of TILs in favor of tumor immune evasion.

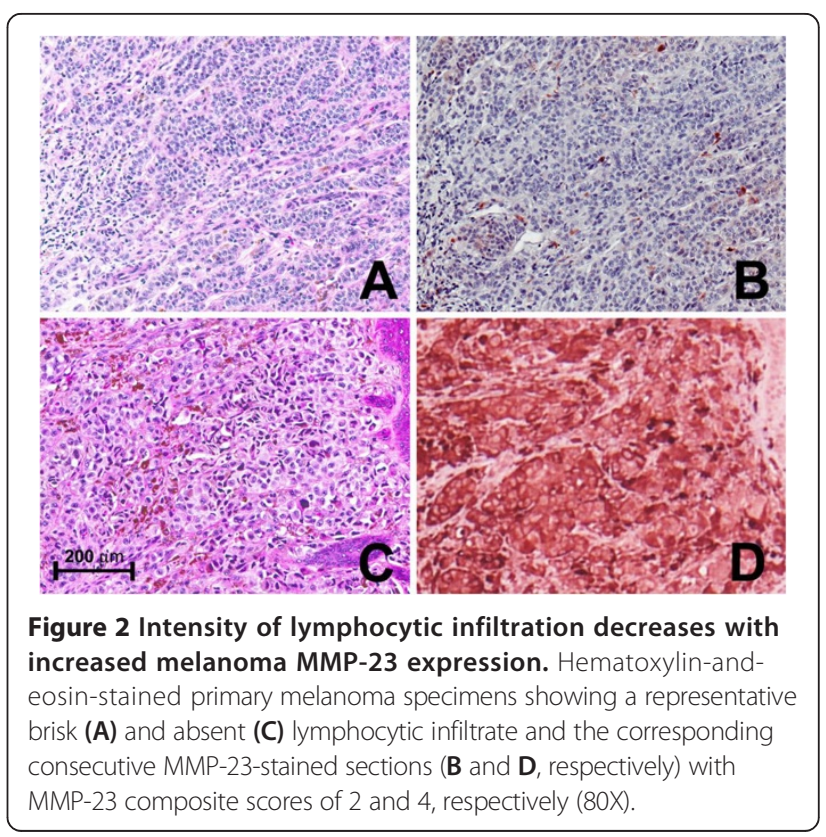




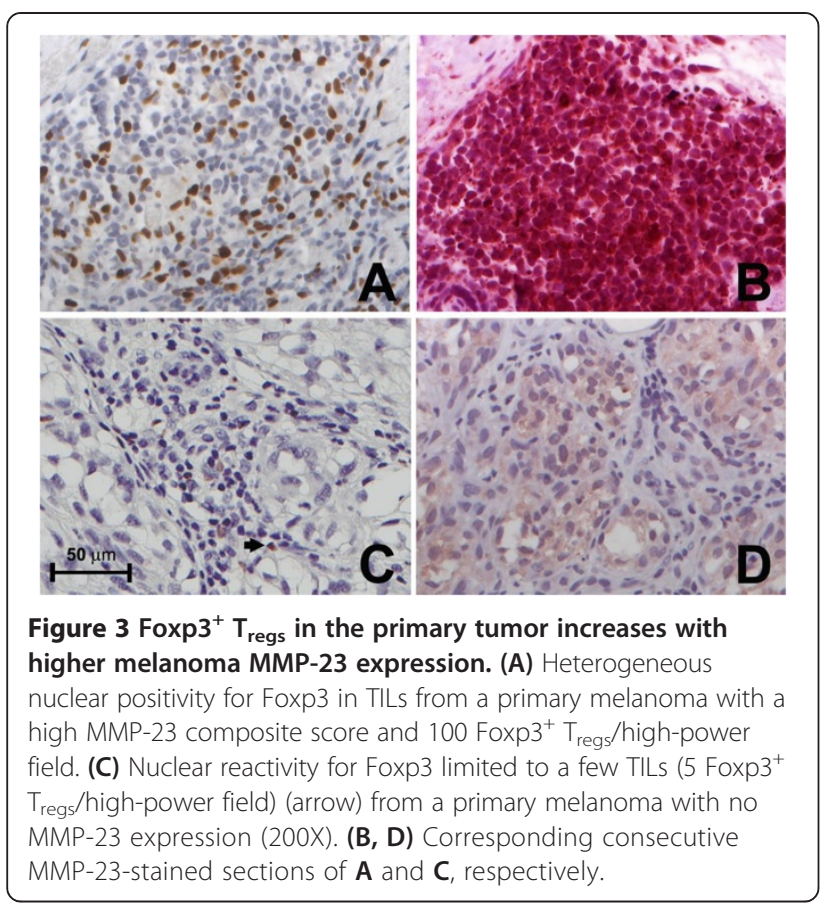

Higher MMP-23 expression is associated with resistance to immune biologic immunotherapy

To further evaluate the potential role of MMP-23 in tumor immune escape, we examined the relationship between primary melanoma MMP-23 expression and clinical response to immunotherapy, as measured by the rate of recurrence. Considering the entire cohort of patients - receiving immunotherapy after primary resection or at recurrence subsequent recurrence was detected in 11/19 (59\%) patients with low melanoma MMP-23 expression (composite score $=0-2$ ) compared with $42 / 52$ (81\%) patients high melanoma MMP-23 expression (composite score = $3-4)(P=0.067)$ (Table 3$)$. This trend holds when considering only patients who had received immunotherapy after primary resection (vaccines $(n=21)$, immune biologics $(n=19))$, where the recurrence rate was lower in patients

Table 3 Analysis of melanoma recurrence according to MMP-23 expression in the entire cohort and the subset of patients who received immunotherapy after primary resection

\begin{tabular}{|c|c|c|c|c|}
\hline & \multicolumn{4}{|c|}{ MMP-23 composite score } \\
\hline & \multicolumn{2}{|c|}{ Entire cohort $^{\mathrm{a}}$} & \multicolumn{2}{|c|}{$\begin{array}{l}\text { Immunotherapy after } \\
\text { primary resection }\end{array}$} \\
\hline & $0-2$ & $3-4$ & $0-2$ & $3-4$ \\
\hline \multicolumn{5}{|c|}{ Recurrence } \\
\hline Yes & $11(59 \%)$ & $42(81 \%)$ & $5(46 \%)$ & $23(79 \%)$ \\
\hline No & $8(41 \%)$ & $10(19 \%)$ & $6(54 \%)$ & $6(21 \%)$ \\
\hline Total & $19(100 \%)$ & $52(100 \%)$ & $11(100 \%)$ & $29(100 \%)$ \\
\hline
\end{tabular}

with low melanoma MMP-23 expression (5/11; 46\%) compared with patients with high melanoma MMP-23 expression $(23 / 29 ; 79 \%)(P=0.056)$ (Table 3$)$. These trends suggest that MMP-23 may play a role in regulating immune responses in the context of immunotherapy.

Different classes of immunotherapies target different components of the anti-tumor immune response. Vaccines target $\mathrm{CD}^{+}{ }^{+} \mathrm{CCR} 7^{+}$central memory $\mathrm{T}$ cells $\left(\mathrm{T}_{\mathrm{CM}}\right)$ and immune biologics target $\mathrm{CD}^{+} \mathrm{CCR}^{-}$effector memory $\mathrm{T}$ cells $\left(\mathrm{T}_{\mathrm{EM}}\right)$ [37-39]. Therefore, we evaluated the clinical outcomes of primary melanoma patients treated with vaccines and immune biologics separately to determine if the observed relationship between tumor MMP-23 expression and recurrence could be attributed to an effect on a specific $\mathrm{T}$ cell subset (Table 4). Considering all patients who received vaccine therapy (after primary resection or at recurrence), no significant difference in recurrence was detected between patients with low melanoma MMP-23 expression and those with high melanoma MMP-23 expression. This is also true when considering all patients who received immune biologics (after primary resection or at recurrence). However, when we focused our analysis on patients treated with immune biologics only after primary resection, and therefore as primary adjuvant therapy, higher primary melanoma MMP-23 expression was associated with increased recurrence, as recurrence was detected in $1 / 4$ (25\%) patients with low melanoma MMP-23 expression, compared with 13/15 (87\%) patients with high melanoma MMP-23 expression $(P=0.037)$. In contrast, the level of tumor expression of MMP-23 in patients treated with vaccine immunotherapies at primary resection was not associated with recurrence $(P=0.64)$.

To further investigate the affect of MMP-23 on patients receiving immune biologics, we next compared the progression free survival between patients with high versus low melanoma MMP-23 expression (Figure 4). These data revealed that high melanoma MMP-23 expression is associated with shorter periods of progression-free survival $(P=0.025)$. Together, these results suggest that,

Table 4 Stratified analysis of melanoma recurrence risk by type of immunotherapy (immune biologics and vaccines) in the patients who had primary adjuvant immunotherapy

\begin{tabular}{|c|c|c|c|c|}
\hline & \multicolumn{4}{|c|}{ MMP-23 composite score } \\
\hline & \multicolumn{2}{|c|}{ Immune biologics $^{a}$} & \multicolumn{2}{|c|}{ Vaccines $^{\mathbf{b}}$} \\
\hline & $0-2$ & $3-4$ & $0-2$ & $3-4$ \\
\hline \multicolumn{5}{|c|}{ Recurrence } \\
\hline Yes & $1(25 \%)$ & $13(87 \%)$ & $4(57 \%)$ & $10(71 \%)$ \\
\hline No & $3(75 \%)$ & $2(23 \%)$ & $3(43 \%)$ & $4(29 \%)$ \\
\hline Total & $4(100 \%)$ & $15(100 \%)$ & $7(100 \%)$ & $14(100 \%)$ \\
\hline
\end{tabular}




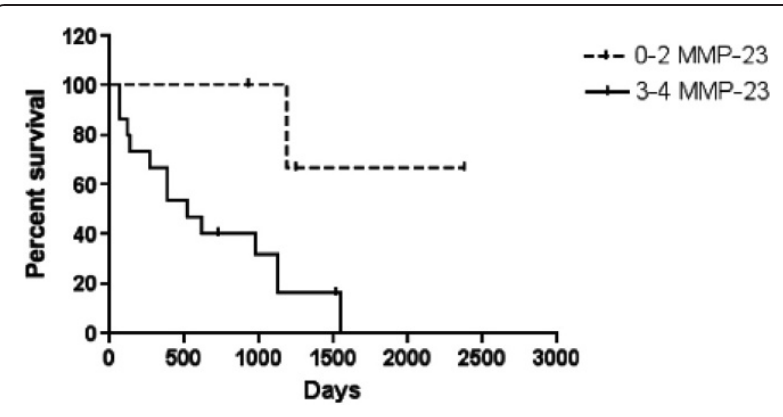

Figure 4 Progression-free survival of melanoma patients receiving immune biologics. Patients treated with immune biologics were monitored for recurrence from the time treatment, and grouped based on expression of MMP-23 prior to initiation of treatment (dashed line - MMP-23 composite score $=0-2$; solid line - MMP-23 composite score $=3-4$ ). $P=0.025$.

in patients treated with immune biologics, high MMP-23 expression levels augment the anti-tumor immune response to both increase the likelihood of recurrence and shorten the time to recurrence. Although statistically significant, we acknowledge that larger sample sizes are required to test these results with greater statistical rigor. These results suggest that high tumor expression of MMP-23 confers a level of resistance to immune biological therapy when given as a primary adjuvant. Interestingly, tumor MMP-23 seems to affect specifically $\mathrm{T}_{\mathrm{EM}}$ cells, as immune biologics including IFN- $\alpha$, IL-2, and GM-CSF function by targeting $\mathrm{T}_{\mathrm{EM}}$ expansion.

\section{Discussion}

We examined the expression of MMP-23 in melanoma and our data suggest that MMP-23 represents an immune escape mechanism and potential immunotherapeutic target. Our data show that melanoma MMP-23 expression correlates with a diminished anti-tumor $\mathrm{T}$ cell response and higher numbers of $\mathrm{T}_{\text {regs }}$ in the TIL population. These data together support a role for tumor MMP-23 in modulating both the infiltration and activation of tumor-reactive lymphocytes.

Anti-tumor $\mathrm{T}$ cells comprise the majority of the tumor lymphocytic infiltrate, and our study shows that the number, intensity of infiltration, and composition of TILs are negatively affected by melanoma MMP-23 expression. Increased tumor expression of MMP-23, therefore, mediates intrinsic tolerance with the potential to confer resistance against immunotherapy. As such, an evaluation of melanoma MMP-23 expression may be particularly useful in selecting candidates for adoptive $\mathrm{T}$ cell therapy, the success of which depends on the harvest, expansion and immunophenotype of TILs [40]. Furthermore, our results suggest the clinical relevance of assessing primary melanoma MMP-23 expression prior to initiating adjuvant treatment with immune biologics, as melanoma MMP-23 expression negatively correlates with response to adjuvant immune biologic therapy. Immune biologics, such as IFN- $\alpha$, IL-2, and GM-CSF, preferentially expand $\mathrm{T}_{\mathrm{EM}}$ cells, whereas vaccines induce $T_{C M}$ responses. The dependence of immune biologics but not vaccines on MMP-23 expression, suggests that MMP-23 specifically affects anti-tumor $\mathrm{T}_{\mathrm{EM}}$ responses. When activated, $\mathrm{T}_{\mathrm{EM}}$ express an increased number of Kv1.3 channels $[37,38]$ and sustained and complete activation is dependent of their function. On the other hand, $\mathrm{T}_{\mathrm{CM}}$ are characterized by low Kv1.3 expression and are not dependent on Kv1.3 function for activation [14]. Therefore, MMP-23 may inhibit anti-tumor $\mathrm{T}_{\mathrm{EM}}$ responses in adjuvant immune biologic therapies through in trans blocking of $\mathrm{T}_{\mathrm{EM}}$ Kv1.3 channels. This could explain the lack of an observed association between melanoma MMP-23 expression and recurrence among patients treated with adjuvant vaccine therapy. Patient stratification based on MMP-23 expression, type of adjuvant therapy, and timing of treatment (i.e. at the time of primary resection) significantly reduced the number of patients in specific groups. The statistical analysis of these data, specifically patients with low MMP-23 expression receiving immune biologic therapy at primary resection $(\mathrm{n}=4)$, is presented with the acknowledgement that the conclusions drawn from these data would benefit from larger sample sizes.

The observed trend toward an increased number of $\mathrm{T}_{\text {regs }}$ within tumors that expressed high levels of MMP23 suggests that this may contribute further to suppression of anti-tumor $\mathrm{T}$ cell responses. A number of studies have reported a correlation between $\mathrm{T}_{\text {reg }}$ infiltration and patient prognosis, where high percentages of $\mathrm{T}_{\text {regs }}$ in both primary melanomas and lymph node metastases correlated with increased recurrence and decreased survival rates [41-43], although this correlation was not observed in other studies $[44,45] . \mathrm{T}_{\text {reg }}$ accumulation in the tumor may be driven by a number of factors, including local chemokine and integrin-ligand expression [46], and immunosuppressive factors promoting expansion of existing $\mathrm{T}_{\text {regs }}$ or generation of induced $\mathrm{T}_{\text {regs }}$ [47]. How increased MMP-23 expression might function to increase the tumor $\mathrm{T}_{\text {reg }}$ population is unclear. Recent work by Godefroy and colleagues [19] showed that MMP-2 in melanoma degrades type I IFN receptor, effectively preventing IL-12p35 production, and skews CD4 T cells towards a Th2 phenotype. It is possible that MMP-23 activity alters signaling pathways controlling $\mathrm{T}_{\text {reg }}$ expansion or de novo production of induced $\mathrm{T}_{\text {regs. }}$ A Kv1.3 knock-out induced EAE mouse model of infection showed that CD4+ T cells expressed greater levels of IL-10 and lower levels of IL-17 and IFN- $\gamma$, and suppressed proliferation of wild-type CD4+ T cells, suggesting a skewing toward a regulatory phenotype [48], although an increase in the number of Foxp3+ cells was not observed. 
While our data suggest a role for melanoma MMP-23 in blunting anti-tumor immunity by selectively blocking T cell Kv1.3 channels, we also explored the possibility that melanoma MMP-23 may act in cis by trapping Kv1.3 in the ER and preventing surface expression. Of the primary melanomas evaluated for Kv1.3 surface expression, most were negative, which may reflect the suppression of Kv1.3 surface expression due to in cis MMP-23 trapping [12] or an absence of Kv1.3 expression altogether. However, for our limited sample size, we did not observe a relationship between MMP-23 expression and tumor Kv1.3 surface staining. Kv1.3 channels on melanoma cells, however, have previously been shown to be in close proximity to $\beta 1$-integrins, such that blockade of Kv1.3 channels dysregulates integrin function and results in loss of cell adherence [31]. Disruption of cell-cell/ cell-matrix adhesion is one of many steps in metastatic progression, and evidence from prostate cancer support the association between reduced tumor cell Kv1.3 expression and poor clinical outcome [32]. Studies in breast and colon cancer, in contrast, suggest that blockade of tumor cell Kv1.3 expression is protective [49,50] as it prevents progression through the $G_{1} / S$ checkpoint, which requires transient hyperpolarization [51]. In trapping Kv1.3 in the ER, melanoma MMP-23 would likewise alter the tumor cell membrane potential and facilitate the transition to the $\mathrm{M}$ phase. Further studies are warranted to explore the possible role of MMP-23 in tumor Kv1.3 suppression.

Inhibition of MMP-23 in combination with other immunotherapies may further augment anti-tumor immunity. With the clinical success of monoclonal antibodies against inhibitory immune checkpoint proteins in melanoma, including CTLA-4 [52] and PD-1 [53], anti-MMP23 therapy also holds promise as a potential treatment strategy. Yet, such strategies must consider the possibility of off-target effects; thus the normal tissue distribution and physiologic role of MMP-23 must first be understood. Unlike other MMPs, MMP-23 is widely expressed under physiologic conditions, in particular at high levels in the ovary, testis, prostate, and heart and at low levels in the lung, pancreas, and colon [10,54]. Intralesional delivery of MMP-23 inhibitors may therefore be preferred over systemic injection to minimize possible off-target effects. Furthermore, locoregional MMP-23 inhibition has the potential to alter both the number and the composition of the TILs, such that adoptive T cell therapy candidates could benefit from pre-treatment with intralesional MMP23 inhibitors.

In identifying MMP-23 as a novel immunotherapeutic target, it is important to recognize that inhibition of MMP-23 may result in immune-related adverse events, much like the inhibition of CTLA-4 [55]. Ongoing efforts to identify biomarkers to predict the response to anti-CTLA-4 therapy are under way just as additional studies to identify those patients most likely to benefit from MMP-23 inhibition are needed in parallel to those of the role of MMP-23 in melanoma. Our study provides the foundation for both as data suggest that an assessment of melanoma MMP-23 expression by routine immunohistochemistry may prove useful, much like using immunohistochemistry to determine the estrogen/progesterone receptor status in breast cancer [56]. Yet, the clinical value of assessing melanoma MMP-23 expression in conjunction with Kv1.3 expression remains to be determined.

\section{Conclusions}

Our study suggests that MMP-23 may play a role in tumor-induced immune escape and that melanoma MMP23 expression represents a novel biomarker and possible immunotherapeutic target. MMP-23 inhibition in combination with other therapeutic agents may be more effective than as monotherapy, yet studies are still needed to elucidate the exact mechanism by which melanomas upregulate MMP-23 to allow the development of a specific MMP-23 inhibitor with a favorable risk-benefit profile.

\section{Additional files}

Additional file 1: Figure S1. Demonstration of MMP-23 antibody specificity by competition with immunizing peptide. Immunostaining by MMP-23 primary antibody plus secondary antibody (A), preincubated with immunizing peptide before addition of primary and secondary antibodies (B), and with secondary antibody only in the absence of primary MMP-23 antibody (C).

Additional file 2: Figure S2. Western blot demonstrating detection of MMP23 expression using MMP-23 antibody. (A) MMP-23 Western blots using different MMP-23-specific antibodies to probe $10 \mu \mathrm{g}$ of placenta protein per lane: Lane A) - ab39087, immunogen C terminus of MMP-23, diluted 1:5000; Lane B) ab74215 immunogen peptide derived from the $C$ terminus of human MMP-23 protein, diluted 1:1000; Lane C) ab39086, immunogen peptide corresponding to the hinge region of human MMP-23, diluted 1:5000; and Lane D) ab 53148, immunogen Synthetic peptide derived from human MMP-23, diluted 1:1000. (B) $10 \mu \mathrm{g}$ of protein was extracted from melanoma tissues (Lanes A-D), melanoma cell line (Lane E) or placenta (Lane F) and assessed by Western blot using ant-MMP-23 antibody, ab39087.

\section{Abbreviations}

MMP-23: Matrix metalloproteinase-23; MMPs: Matrix metalloproteinases; MMP-23-PD: MMP-23 pro-domain; MMP-23-TxD: MMP-23 toxin-like domain; TIL: Tumor infiltrating lymphocytes; CTLA-4: Cytotoxic T lymphocyte-associated antigen-4; PD-1: programmed cell death-1; ECM: Extracellular matrix; ER: Endoplasmic reticulum; $T_{C M}$ : Central memory T cell; $T_{E M}$ : Effector memory T cell.

\section{Competing interests}

The authors have no competing interests to declare. A patent application pertaining to cancer treatment methods and the role of MMP-23 as a therapeutic target (PCT/US13/39022) has been filed by M.K. and I.O.

\section{Authors' contributions}

Conception and design: DM, MWM, AP, IO, MK; Development of methodology: DM, MWM, AP, EBF, EVM, FD, PJC, IO, MK; Acquisition of data: DM, MWM, EBF, EVM, FD, PS, AP, ACP, NB, IO, MK; Analysis and interpretation of data: DM, MWM, IS, EBF, EVM, NB, PJC, IO, MK; Writing, review, and/or revision of the manuscript: DM, MWM, IS, EBF, EVM, FD, PS, AP, ACP, NB, PJC, IO, MK; Administrative, technical, or material support: PS, IO, MK; Study 
supervision: IO, MK; Review of pathologic samples: FD All authors read and approved the final manuscript.

\section{Acknowledgements}

We thank Dr. Edward Skolnik and Dr. Stefan Feske for their critical reading of the manuscript. M.K. was a Pew Scholar in the Biomedical Sciences supported by the Pew Trust. This work was supported by the National Institute of Health grants NCI 1U01CA137070 and NIGMS 5R01GM085586 (to M.K.), an American Cancer Society Research Scholar grant RSG-09-070-01-LIB (to M.K), a Cancer Research Investigator grant from the Cancer Research Institute (CRI) (to M.K.) and the NYU Cancer Institute, the NYU Cancer Institute Cancer Center Support Grant 5P30CA016087-27 (to I.O.), and the Marc Jacobs campaign to support the Interdisciplinary Melanoma Cooperative Group.

\section{Author details}

${ }^{1}$ Perlmutter Cancer Center at NYU Langone, New York, NY, USA. ²Department of Pathology, New York University School of Medicine, New York, NY, USA. ${ }^{3}$ Ronald O. Perelman Department of Dermatology, New York University School of Medicine, New York, NY, USA. ${ }^{4}$ Interdisciplinary Melanoma Cooperative Group, New York University School of Medicine, New York, NY, USA. Instituto Português de Oncologia de Lisboa Francisco Gentil, Lisboa, Portugal. ${ }^{6}$ Programme for Advanced Medical Education, Lisbon, Portugal. ${ }^{7}$ Department of Surgery, New York University School of Medicine, New York, NY, USA. ${ }^{8}$ Department of Medicine, New York University School of Medicine, New York, NY, USA. ${ }^{9}$ Division of Biostatistics and Epidemiology, Weill Cornell Medical College, New York, NY, USA.

Received: 24 July 2014 Accepted: 24 November 2014

Published online: 10 December 2014

\section{References}

1. Alexandrescu DT, Ichim TE, Riordan NH, Marincola FM, Di Nardo A, Kabigting FD, Dasanu CA: Immunotherapy for melanoma: current status and perspectives. J Immunother 2010, 33:570-590.

2. Loose D, Van de Wiele C: The immune system and cancer. Cancer Biother Radiopharm 2009, 24:369-376.

3. Frey AB, Monu N: Signaling defects in anti-tumor T cells. Immunol Rev 2008, 222:192-205

4. Galea CA, Nguyen HM, George Chandy K, Smith BJ, Norton RS: Domain structure and function of matrix metalloprotease 23 (MMP23): role in potassium channel trafficking. CMLS: Cellular and molecular life sciences; 2013

5. Butler GS, Overall CM: Updated biological roles for matrix metalloproteinases and new "intracellular" substrates revealed by degradomics. Biochemistry 2009, 48:10830-10845.

6. Shuman Moss LA, Jensen-Taubman S, Stetler-Stevenson WG: Matrix metalloproteinases: changing roles in tumor progression and metastasis. Am J Pathol 2012, 181:1895-1899.

7. Hadler-Olsen E, Fadnes B, Sylte I, Uhlin-Hansen L, Winberg JO: Regulation of matrix metalloproteinase activity in health and disease. FEBS J 2011, 278:28-45.

8. Sbardella D, Fasciglione GF, Gioia M, Ciaccio C, Tundo GR, Marini S, Coletta M: Human matrix metalloproteinases: an ubiquitarian class of enzymes involved in several pathological processes. Mol Asp Med 2012, 33:119-208.

9. Ohnishi J, Ohnishi E, Jin M, Hirano W, Nakane D, Matsui H, Kimura A, Sawa H, Nakayama K, Shibuya H, Nagashima K, Takahashi T: Cloning and characterization of a rat ortholog of MMP-23 (matrix metalloproteinase-23), a unique type of membrane-anchored matrix metalloproteinase and conditioned switching of its expression during the ovarian follicular development. Mol Endocrinol 2001, 15:747-764.

10. Pei D, Kang T, Qi H: Cysteine array matrix metalloproteinase (CA-MMP)/ MMP-23 is a type II transmembrane matrix metalloproteinase regulated by a single cleavage for both secretion and activation. J Biol Chem 2000, 275:33988-33997.

11. Nguyen HM, Galea CA, Schmunk G, Smith BJ, Edwards RA, Norton RS, Chandy KG: Intracellular trafficking of the KV1.3 potassium channel is regulated by the prodomain of a matrix metalloprotease. J Biol Chem 2013, 288:6451-6464.

12. Rangaraju S, Khoo KK, Feng ZP, Crossley G, Nugent D, Khaytin I, Chi V, Pham C, Calabresi P, Pennington MW, Norton RS, Chandy KG: Potassium channel modulation by a toxin domain in matrix metalloprotease 23. $J$ Biol Chem 2010, 285:9124-9136

13. Beeton C, Wulff $\mathrm{H}$, Standifer NE, Azam P, Mullen KM, Pennington MW, Kolski-Andreaco A, Wei E, Grino A, Counts DR, Wang PH, LeeHealey CJ BSA, Sankaranarayanan A, Homerick D, Roeck WW, Tehranzadeh J, Stanhope KL, Zimin P, Havel PJ, Griffey S, Knaus HG, Nepom GT, Gutman GA, Calabresi PA Chandy KG: Kv1.3 channels are a therapeutic target for T cell-mediated autoimmune diseases. Proc Nat Acad Sci USA 2006, 103:17414-17419.

14. Rangaraju S, Chi V, Pennington MW, Chandy KG: Kv1.3 potassium channels as a therapeutic target in multiple sclerosis. Expert Opin Ther Targets 2009, 13:909-924.

15. Hofmann UB, Westphal JR, Van Muijen GN, Ruiter DJ: Matrix metalloproteinases in human melanoma. J Investigative Derm 2000, 115:337-344.

16. Egeblad M, Werb Z: New functions for the matrix metalloproteinases in cancer progression. Nat Rev Cancer 2002, 2:161-174.

17. Roy R, Yang J, Moses MA: Matrix metalloproteinases as novel biomarkers and potential therapeutic targets in human cancer. J Clin Oncol 2009, 27:5287-5297

18. Grivennikov SI, Greten FR, Karin M: Immunity, inflammation, and cancer. Cell 2010, 140:883-899.

19. Godefroy E, Manches O, Dreno B, Hochman T, Rolnitzky L, Labarriere N, Guilloux Y, Goldberg J, Jotereau F, Bhardwaj N: Matrix metalloproteinase-2 conditions human dendritic cells to prime inflammatory $\mathrm{T}(\mathrm{H}) 2$ cells via an IL-12- and OX40L-dependent pathway. Cancer Cell 2011, 19:333-346.

20. Li M, Xiao T, Zhang Y, Feng L, Lin D, Liu Y, Mao Y, Guo S, Han N, Di X, Zhang K, Cheng S, Gao Y: Prognostic significance of matrix metalloproteinase-1 levels in peripheral plasma and tumour tissues of lung cancer patients. Lung Cancer 2010, 69:341-347.

21. Qian Q, Wang Q, Zhan P, Peng L, Wei SZ, Shi Y, Song Y: The role of matrix metalloproteinase 2 on the survival of patients with non-small cell lung cancer: a systematic review with meta-analysis. Cancer Investig 2010, 28:661-669.

22. Liu D, Nakano J, Ishikawa S, Yokomise H, Ueno M, Kadota K, Urushihara M, Huang CL: Overexpression of matrix metalloproteinase-7 (MMP-7) correlates with tumor proliferation, and a poor prognosis in non-small cell lung cancer. Lung Cancer 2007, 58:384-391.

23. McGowan PM, Duffy MJ: Matrix metalloproteinase expression and outcome in patients with breast cancer: analysis of a published database. Ann Oncol 2008, 19:1566-1572.

24. Mylona E, Nomikos A, Magkou C, Kamberou M, Papassideri I, Keramopoulos A, Nakopoulou L: The clinicopathological and prognostic significance of membrane type 1 matrix metalloproteinase (MT1-MMP) and MMP-9 according to their localization in invasive breast carcinoma. Histopathology 2007, 50:338-347.

25. Hofmann UB, Westphal JR, Waas ET, Zendman AJ, Cornelissen IM, Ruiter DJ, van Muijen GN: Matrix metalloproteinases in human melanoma cell lines and xenografts: increased expression of activated matrix metalloproteinase-2 (MMP-2) correlates with melanoma progression. $\mathrm{Br} J$ Cancer 1999, 81:774-782

26. Redondo $P$, Lloret $P$, Idoate $M$, Inoges $S$ : Expression and serum levels of MMP-2 and MMP-9 during human melanoma progression. Clin Exp Dermatol 2005, 30:541-545.

27. Candrea E, Senila S, Tatomir C, Cosgarea R: Active and inactive forms of matrix metalloproteinases 2 and 9 in cutaneous melanoma. Int $J$ Dermatol 2014, 53:575-580

28. Coussens LM, Fingleton B, Matrisian LM: Matrix metalloproteinase inhibitors and cancer: trials and tribulations. Science 2002, 295:2387-2392.

29. Wich LG, Hamilton HK, Shapiro RL, Pavlick A, Berman RS, Polsky D, Goldberg JD, Hernando E, Manga P, Krogsgaard M, Kamino H, Darvishian F, Lee P, Orlow SJ, Ostrer H, Bhardwaj N, Osman I: Developing a multidisciplinary prospective melanoma biospecimen repository to advance translational research. Am J Transl Res 2009, 1:35-43.

30. Clark WH Jr, Elder DE, Guerry D, Braitman LE, Trock BJ, Schultz D, Synnestvedt M, Halpern AC: Model predicting survival in stage I melanoma based on tumor progression. J Natl Cancer Inst 1989, 81:1893-1904.

31. Artym W, Petty HR: Molecular proximity of Kv1.3 voltage-gated potassium channels and beta(1)-integrins on the plasma membrane of melanoma cells: effects of cell adherence and channel blockers. J Gen Physiol 2002, 120:29-37.

32. Abdul M, Hoosein N: Reduced Kv1.3 potassium channel expression in human prostate cancer. J Membrane Biology 2006, 214:99-102. 
33. Matheu MP, Beeton C, Garcia A, Chi V, Rangaraju S, Safrina O, Monaghan K, Uemura MI, Li D, Pal S, de la Maza LM, Monuki E, Flugel A, Pennington MW, Parker I, Chandy KG, Cahalan MD: Imaging of effector memory T cells during a delayed-type hypersensitivity reaction and suppression by Kv1.3 channel block. Immunity 2008, 29:602-614.

34. Oble DA, Loewe R, Yu P, Mihm MC Jr: Focus on TILs: prognostic significance of tumor infiltrating lymphocytes in human melanoma. Cancer Immun 2009, 9:3.

35. De Panfilis G, Campanini N, Santini M, Mori G, Tognetti E, Maestri R, Lombardi M, Froio E, Ferrari D, Ricci R: Phase- and stage-related proportions of $T$ cells bearing the transcription factor FOXP3 infiltrate primary melanoma. J Investigative Derma 2008, 128:676-684.

36. Jacobs JF, Nierkens S, Figdor CG, de Vries IJ, Adema GJ: Regulatory T cells in melanoma: the final hurdle towards effective immunotherapy? lancet Oncol 2012, 13:e32-e42.

37. Sikora AG, Jaffarzad N, Hailemichael Y, Gelbard A, Stonier SW, Schluns KS, Frasca L, Lou Y, Liu C, Andersson HA, Hwu P, Overwijk WW: IFN-alpha enhances peptide vaccine-induced CD8+ T cell numbers, effector function, and antitumor activity. J Immunol 2009, 182:7398-7407.

38. Hueman MT, Stojadinovic A, Storrer CE, Dehqanzada ZA, Gurney JM, Shriver CD, Ponniah S, Peoples GE: Analysis of naive and memory CD4 and CD8 T cell populations in breast cancer patients receiving a HER2/neu peptide (E75) and GM-CSF vaccine. Cancer Immun, Immunotherapy 2007, 56:135-146.

39. Butler NS, Nolz JC, Harty JT: Immunologic considerations for generating memory CD8 T cells through vaccination. Cell Microbiol 2011, 13:925-933.

40. June $\mathrm{CH}$ : Adoptive $\mathrm{T}$ cell therapy for cancer in the clinic. J Clin Invest 2007, 117:1466-1476.

41. Brody JR, Costantino CL, Berger AC, Sato T, Lisanti MP, Yeo CJ, Emmons RV Witkiewicz AK: Expression of indoleamine 2,3-dioxygenase in metastatic malignant melanoma recruits regulatory $T$ cells to avoid immune detection and affects survival. Cell Cycle 2009, 8:1930-1934.

42. Knol AC, Nguyen JM, Quereux G, Brocard A, Khammari A, Dreno B: Prognostic value of tumor-infiltrating Foxp3+ T-cell subpopulations in metastatic melanoma. Exp Dermatol 2011, 20:430-434.

43. Miracco C, Mourmouras V, Biagioli M, Rubegni P, Mannucci S, Monciatti I, Cosci E, Tosi P, Luzi P: Utility of tumour-infiltrating CD25 + FOXP3+ regulatory $T$ cell evaluation in predicting local recurrence in vertical growth phase cutaneous melanoma. Oncol Rep 2007, 18:1115-1122.

44. Ladanyi A, Mohos A, Somlai B, Liszkay G, Gilde K, Fejos Z, Gaudi I, Timar J: FOXP3+ cell density in primary tumor has no prognostic impact in patients with cutaneous malignant melanoma. Pathol Oncol Res 2010, 16:303-309.

45. Hillen F, Baeten $\mathrm{Cl}$, van de Winkel A, Creytens D, van der Schaft DW, Winnepenninckx $V$, Griffioen AW: Leukocyte infiltration and tumor cell plasticity are parameters of aggressiveness in primary cutaneous melanoma. Cancer Immun, Immunotherapy 2008, 57:97-106.

46. Wei S, Kryczek I, Zou W: Regulatory T-cell compartmentalization and trafficking. Blood 2006, 108:426-431.

47. Nizar S, Meyer B, Galustian C, Kumar D, Dalgleish A: T regulatory cells, the evolution of targeted immunotherapy. Biochim Biophys Acta 2010, 1806:7-17.

48. Gocke AR, Lebson LA, Grishkan IV, Hu L, Nguyen HM, Whartenby KA Chandy KG, Calabresi PA: Kv1.3 deletion biases T cells toward an immunoregulatory phenotype and renders mice resistant to autoimmune encephalomyelitis. J Immunol 2012, 188:5877-5886.

49. Abdul M, Santo A, Hoosein N: Activity of potassium channel-blockers in breast cancer. Anticancer Res 2003, 23:3347-3351.

50. Abdul M, Hoosein N: Voltage-gated potassium ion channels in colon cancer. Oncol Rep 2002, 9:961-964.

51. Blackiston DJ, McLaughlin KA, Levin M: Bioelectric controls of cell proliferation: ion channels, membrane voltage and the cell cycle. Cell Cycle 2009, 8:3519-3528

52. Peggs KS, Quezada SA, Chambers CA, Korman AJ, Allison JP: Blockade of CTLA-4 on both effector and regulatory $T$ cell compartments contributes to the antitumor activity of anti-CTLA-4 antibodies. J Exp Med 2009, 206:1717-1725.

53. Weber J: Immunotherapy for melanoma. Curr Opin Oncol 2011, 23:163-169.

54. Velasco G, Pendas AM, Fueyo A, Knauper V, Murphy G, Lopez-Otin C: Cloning and characterization of human MMP-23, a new matrix metalloproteinase predominantly expressed in reproductive tissues and lacking conserved domains in other family members. J Biol Chem 1999, 274:4570-4576.
55. Hodi FS, O'Day SJ, McDermott DF, Weber RW, Sosman JA, Haanen JB, Gonzalez R, Robert C, Schadendorf D, Hassel JC, Akerley W, van den Eertwegh AJ, Lutzky J, Lorigan P, Vaubel JM, Linette GP, Hogg D, Ottensmeier CH, Lebbe C, Peschel C, Quirt I, Clark JI, Wolchok JD, Weber JS, Tian J, Yellin MJ, Nichol GM, Hoos A, Urba WJ: Improved survival with ipilimumab in patients with metastatic melanoma. N Engl J Med 2010, 363:711-723.

56. Allred DC, Carlson RW, Berry DA, Burstein HJ, Edge SB, Goldstein LJ, Gown A, Hammond ME, Iglehart JD, Moench S, Pierce LJ, Ravdin P, Schnitt SJ, Wolff AC: NCCN Task Force Report: Estrogen Receptor and Progesterone Receptor Testing in Breast Cancer by Immunohistochemistry. JNCCN 2009, 7(Suppl 6):S1-S21. quiz S22-23.

doi:10.1186/s12967-014-0342-7

Cite this article as: Moogk et al:: Melanoma expression of matrix metalloproteinase-23 is associated with blunted tumor immunity and poor responses to immunotherapy. Journal of Translational Medicine 2014 12:342.

\section{Submit your next manuscript to BioMed Central and take full advantage of:}

- Convenient online submission

- Thorough peer review

- No space constraints or color figure charges

- Immediate publication on acceptance

- Inclusion in PubMed, CAS, Scopus and Google Scholar

- Research which is freely available for redistribution 